\title{
ROLE OF CANNABINOID 2 RECEPTOR ACTIVITY IN POST CNS INJURY IMMUNODEFICIENCY
}

\author{
Ian Burkovskiy 1,2, Vladimir Cerny ${ }^{1,5}$, Juan Zhou ${ }^{1}$, Christian Lehmann ${ }^{1,2,3,4}$ \\ 1 Department of Anesthesia, Pain Management and Perioperative Medicine, \\ 2 Department of Pharmacology, ${ }^{3}$ Department of Microbiology \& Immunology, \\ ${ }^{4}$ Department of Physiology \& Biophysics \\ Dalhousie University, Halifax, Nova Scotia, Canada \\ 5 Department of Anesthesiology, Perioperative Medicine and Intensive Care \\ J.E. Purkinje University, Masaryk Hospital, Usti nad Labem, Czech Republic
}

DALHOUSIE

UNIVERSITY

\section{BACKGROUND}

* Patients after acute central nervous system (CNS) injury have a high risk for an infection due to post-injury disturbance of the normally well-balanced interplay between the immune system and the CNS resulting in systemic immunosuppression, as shown in Figure 1 below.

* The endocannabinoid system (ECS) is composed of endocannabinoids, specific receptors and metabolizing enzymes, and is involved in key homeostatic functions in the CNS and the immune system

* The cannabinoid 2 receptor (CB2R) on immune cells and microglia presents a potential therapeutic target as activation of this receptor has been shown to be involved in immunosuppression.

* Our study presents experimental evidence that targeting the CB2R may have beneficial effects on the outcome after an acute CNS injury

* The main objective of the study was to investigate if post CNS injury immunodeficiency can be reversed by - (1) early activation of CB2R using a synthetic agonist HU308 (2.5 mg/kg, i.v.); (2) late deactivation of CB2R using a synthetic CB2R antagonist AM630 (2.5 mg/kg, i.v.)

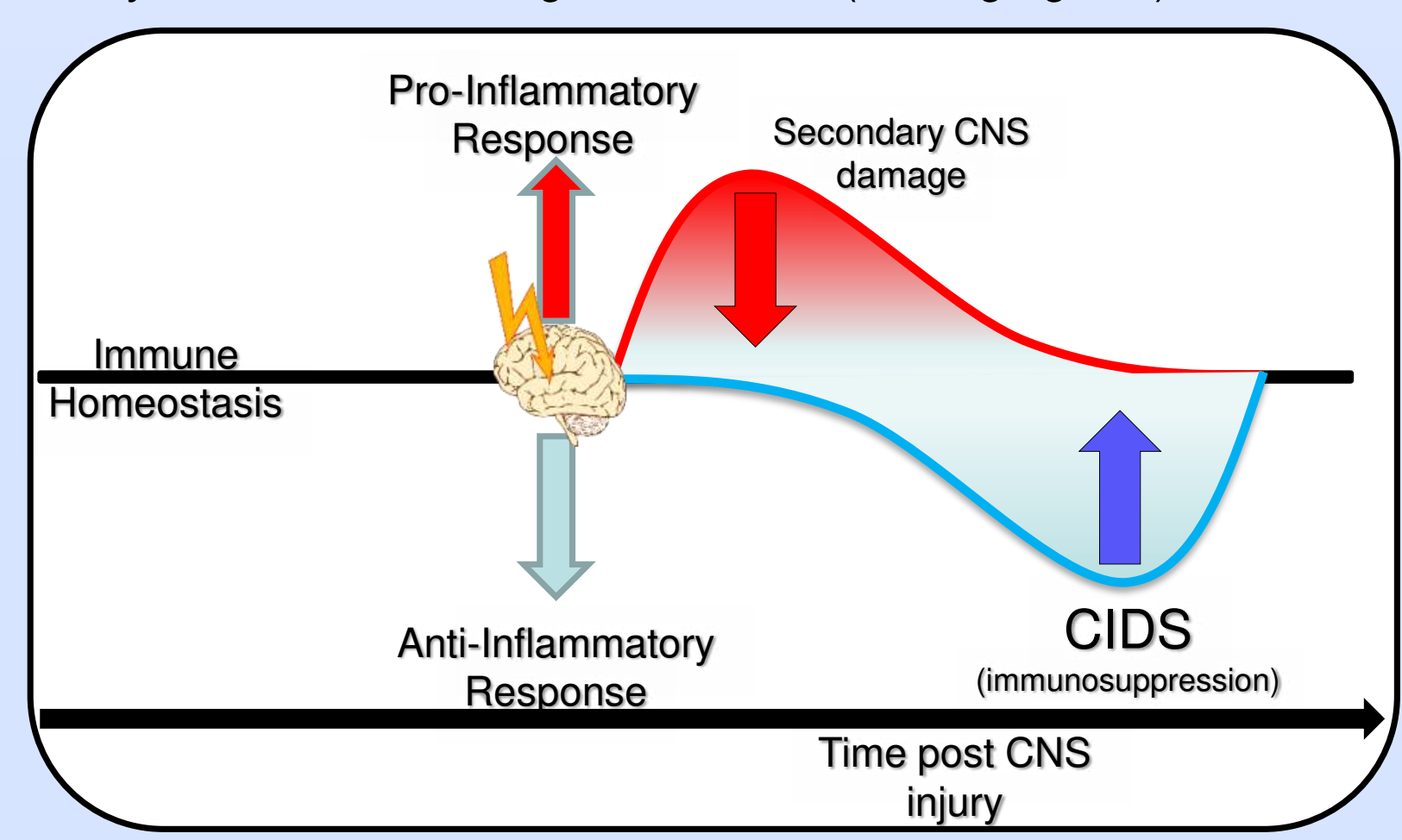

Fiqure 1. Post CNS injury Inflammatory Response

\section{MATERIALS \& METHODS}

- Species: Male C57BL/6 w.t. and CB2R K.O. mice (6-8 weeks). All drugs were administered intravenously.

* CNS Injury Model: CNS injury was induced via two intracerebral injections of a vasoconstrictor peptide,

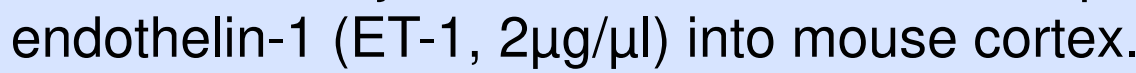

* Microscopy: The extent of immune response to endotoxin (LPS) was assessed with intravital microscopy (IVM) of gut microvasculature. Video sequences were recorded and the extent of leukocyte activation was quantified.

* Staining: Brains of the animals were extracted post mortem and stained with tetrazolium chloride (TTC) to histologically confirm CNS injury and measure infarct size.
RESULTS

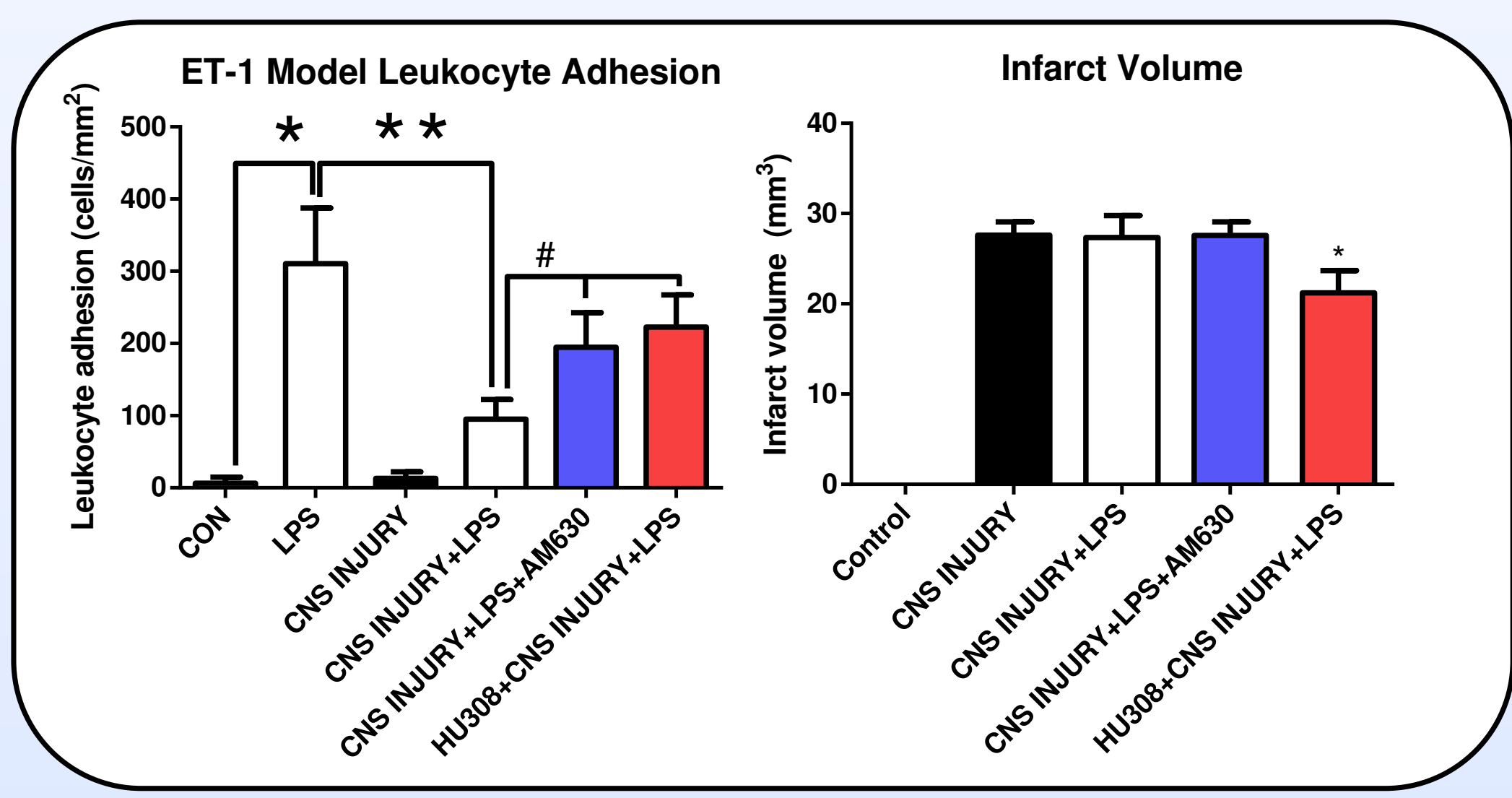

Figure 2. (A) Leukocyte adhesion in collecting venules (V1); ${ }^{*} \mathrm{p}<0.05$ vs CON, ** $p<0.05$ vs LPS, \# $p<0.05$ vs CNS INJURY+LPS (B) Infarct volume, as measured after TTC stain ${ }^{*} \mathrm{p}<0.05$ vs CNS INJURY

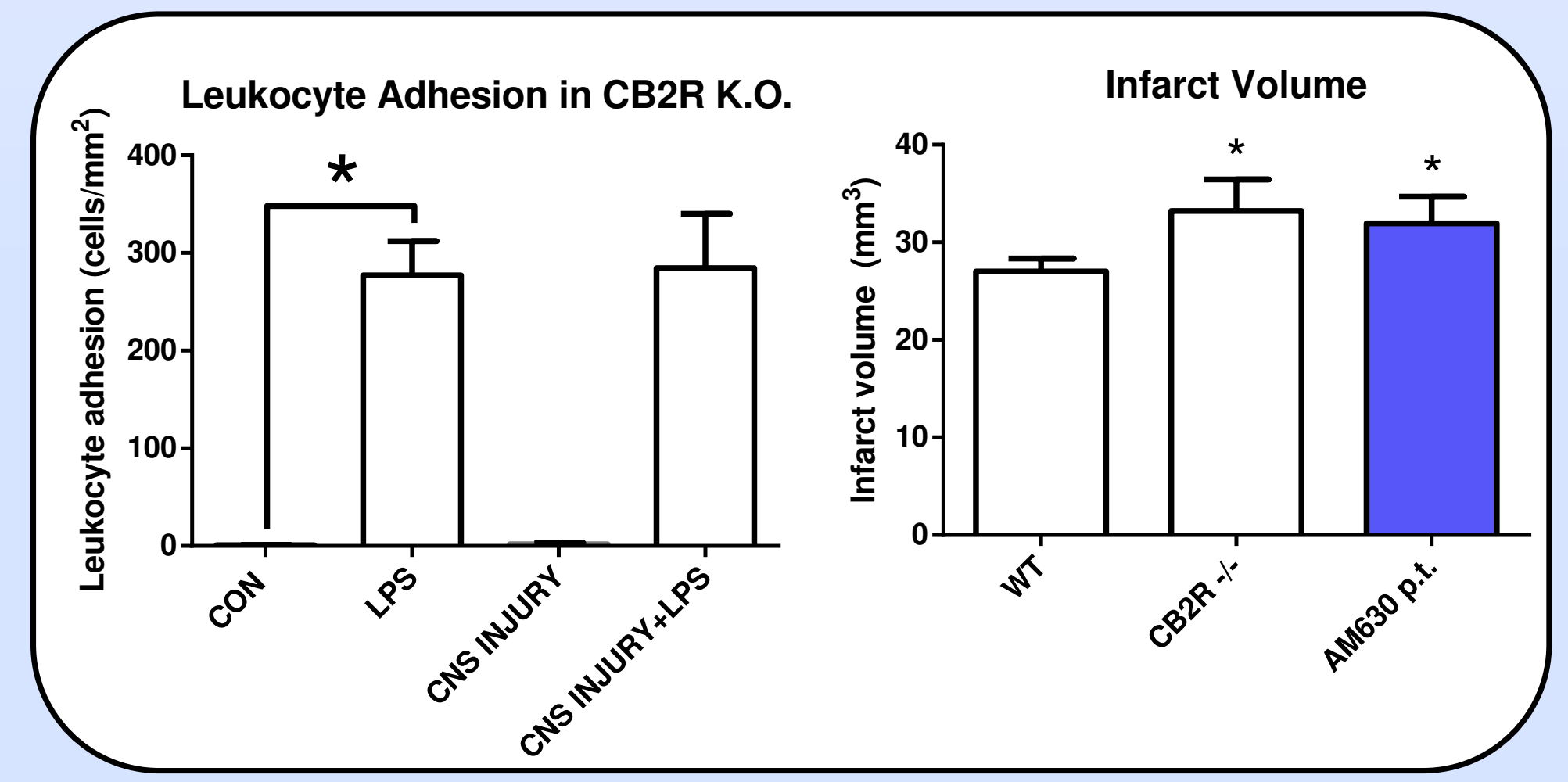

Figure 3. (A) Leukocyte adhesion in collecting venules (V1) in CB2R K.O. mice; ${ }^{*} \mathrm{p}<0.05$ vs $\mathrm{CON}$, (B) Infarct volume in CB2R K.O. mice, as measured after TTC stain ${ }^{*} \mathrm{p}<0.05$ vs WT

\section{CONCLUSION}

* Early CB2R activation attenuates infarct size after CNS injury

* Early CB2R activation reduces the severity of the immune suppression after CNS injury

* Late CB2R inhibition reduces the immune suppression after CNS injury

* CB2R-related modulation of leukocyte activation is involved in the impaired immune response following CNS injury

\section{ACKNOWLEDGEMENTS}

